\title{
Prototype of a controllable damper based on granular materials subjected to partial vacuum
}

\author{
Robert Zalewski ${ }^{1, *}$, Piotr Bartkowski ${ }^{1}$ \\ ${ }^{1}$ Institute of Machines Design Fundamentals, Warsaw University of Technology, Narbutta \\ 84, 02-254 Warsaw, Poland
}

\begin{abstract}
In the paper a proposal of granular damper prototype has been introduced. The granular damper works on the basis of a core made of Vacuum Packed Particles. Idea of Vacuum Packed Particles consists of placing loose granular grains into a hermetic envelope, wherein in the next stage a partial vacuum is generated. The difference between an atmospheric pressure, externally surrounding the core and internal one, causes occurring friction forces between single grains. Interacting friction forces are greater for higher values of internal underpressure. In presented paper the authors focus on analysis of experimental results obtained for various values of underpressure. Variations in energy dissipation possibilities are particularly described in this paper.
\end{abstract}

Keywords: Vacuum Packed Particles, Smart Materials

\section{Introduction}

Controlled damping of noise and vibration is still the object of interest for engineers and researchers. A breakthrough in this field was the end of the last century, when material engineering and information systems progressed, enabling the commercial use of materials belonging to the so-called, group of "smart structures" [1]. From that moment, the research on the possibility of eliminating vibrations harmful to the structure or its user is bi-directional. The first one is the development of new technologies for the production of commercial "intelligent materials" and parallel work on the miniaturization and broadly understood modernization of electronic systems, necessary in the process of controlling their physical parameters. The second, more innovative, is the search for new "materials" whose mechanical properties can be controlled depending on changing and often unpredictable external conditions.

This paper presents the concept of a vibration damper prototype with variable dissipative properties, working on the basis of a core, made of Vacuum Packed Particles (VPP). These structures are built of granular materials (loose grains) placed in an airtight plastomer encapsulation in which a partial vacuum is generated $[2,3]$. The undepressure value create the friction forces between the individual grains and as a result of the whole system [4]. These forces are all the greater, the lower the internal pressure is generated inside the core [5].

\footnotetext{
*Corresponding author: robertzalewski@,wp.pl

Reviewers: Juraj Gerlici, Marek Macko
} 
Keeping in mind the obvious restrictions limited by values of atmospheric pressure and technical vacuum, the underpressure parameter allows a free and smooth change of the global physical properties of Vacuum Packed Particles [6].

In the paper particular attention was paid to the analysis of experimental results obtained for the family of granulated cores filled with polypropylene grains. The cyclic loading of cores by means of kinematic excitation was taken into account. The tests were carried out for three various excitation frequencies and a stepwise changeable, full range of possible underpressures. In addition, attention was paid to the basic problems encountered during the research and ways to eliminate them in the proposed solution of the VPP damper prototype.

\section{Experiments}

Because the basic structural element of the granular damper is the mentioned core, the research part of this work focuses on the analysis of the experimental results of a cylindrical sample filled with loose material. Fig. 1 schematically shows the test sample of the granular core. The sample consisted of two rigid steel discs connected together by a tight and soft plastomer encapsulation. Special handles were attached to the discs, enabling assembly of the granulated core in the jaws of the testing machine. Detailed information on the implementation scheme of a single measurement cycle can be found in paper [7]. It is worth mentioning that in order to develop standards for testing samples filled with granular material, placed in airtight space with underpressure, it was necessary to conduct a number of basic research. Fundamental experiments for Vacuum Packed Particles specimens included uniaxial tensile and compression tests. On their basis, it was possible to estimate the impact of technological parameters of the investigated conglomerates, such as the type of granular filler, the degree of filling the sample with loose material (porosity factor) and the estimation of the so-called 'scale effect' (the influence of the volume of the tested samples on the basic strength parameters). Since the analysis of the results of basic research was the subject matter of the authors previous works [8] in the presented study it was limited to the necessary minimum. However, the attention should be paid to fundamental conclusions resulting from the previous research. Briefly, they can be expressed as follows:

- granulated cores (understood as a tight plastomer coating that limits loose granules and a partial vacuum created inside it - underpressure) behave like typical viscoplastic bodies, - the underpressure parameter is a convenient factor allowing for continuous (to a certain limited extent) control of basic mechanical parameters of cores,

- VPP behave differently depending on the experimentally applied load direction.

The research on cyclic loading of granular samples was carried out on the MTS 809 tensile testing machine (Fig. 2). To eliminate the forces resulting from the inertia of the sample elements, a triangular type of kinematic excitation was applied (Fig. 3). The experiments were carried out with three various frequencies: $0.02 ; 0.25$ and $1.8 \mathrm{~Hz}$. Typical experimental results are depicted in Figs. 4-7. Fig. 4 illustrates the character of the response of a polypropylene grains placed in a cylindrical encapsulation with a diameter of $55 \mathrm{~mm}$ and internal underpressure $p=0.04 \mathrm{MPa}$. Assuming the triangular kinematic excitation $x=f(t)$, the corresponding course of the experimentally recorded values of forces was presented.

At this stage of the data analysis, the asymmetry of the force characteristic as a function of time is visible $(F=f(t))$. Lower forces values are necessary to extend the granulated core by the experimentally determined value of elongation than to compress it to the analogical sample shortening. This phenomenon is more clearly visible in Fig. 5. The stress-strain characteristics of the considered sample is apparently asymmetrical. Transfers to 
dimensionless units (stress-strain) were made to allow comparison of experimental results of core samples with various volumes. A detailed description of the transformation process of measurement coordinates and the assumptions made during its implementation can be found in [9]. The most important, from the point of view of potential engineering applications, for the controllable vibration damper prototype, are the characteristics for cores made of VPP illustrating the effect of the underpressure on the dissipative capabilities of conglomerates. Exemplary experimental results are shown in Fig. 6. Based on the data illustrated in Fig. 6, we note that the effect of the underpressure parameter is unquestionable. A change in the partial vacuum value from $p 1=0.01 \mathrm{MPa}$ to $p 2=0.09 \mathrm{MPa}$ results in an increase in the recorded maximum force by several hundred percent. As already mentioned, special granular structures behave in a macroscopic manner like typical viscoplastic bodies. In this work, an attention is paid to the influence of the kinematic excitation rate (deformation velocity) on the nature of the response of the studied granular cores. Exemplary experimental results are illustrated in Fig. 7.

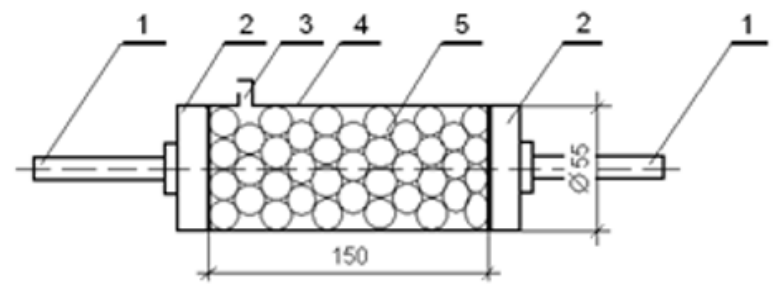

Fig. 1. Scheme of an experimental sample (1-handles, 2-rigid discs, 3-valve, 4-plastomer envelope, 5-grains)

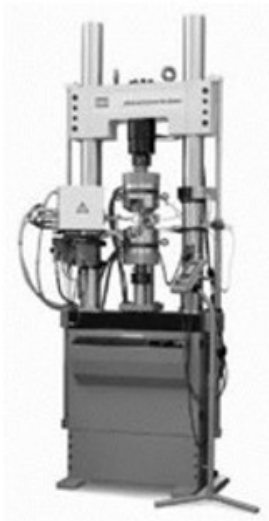

Fig. 2. MTS 809 Universal testing machine 


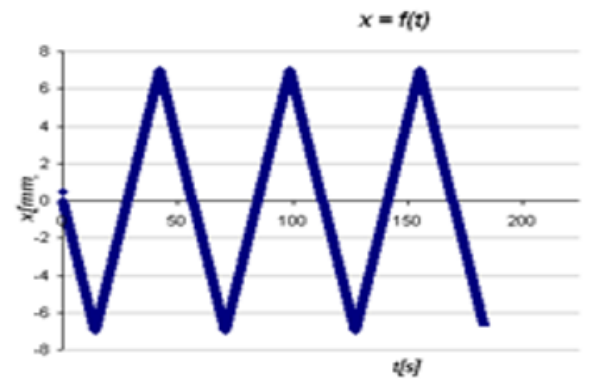

Fig. 3. Kinematical excitation

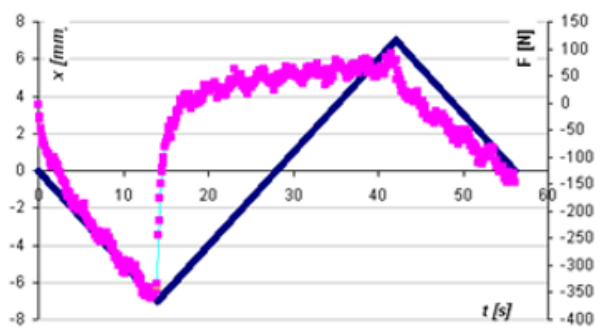

Fig. 4. Typical experimental data: $x=f(t)$ and $F=f(t)$ (material: polypropylene, sample diameter: $55 \mathrm{~mm}$, underpressure: $0.04 \mathrm{MPa}$, excitation frequency: $0.02 \mathrm{~Hz}$ )

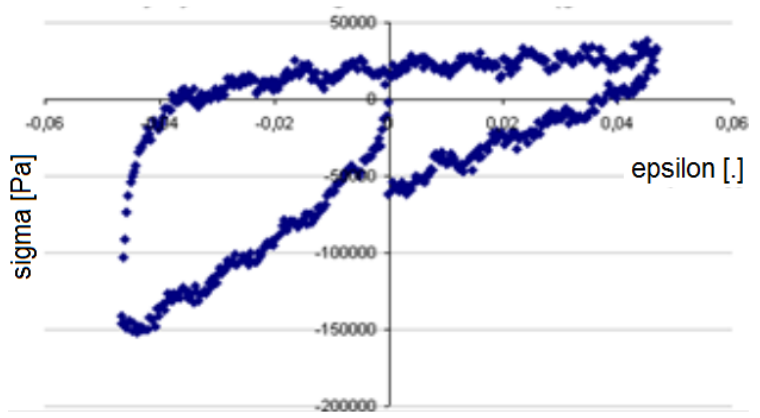

Fig. 5. Typical cyclic loading loop (material: polypropylene, sample diameter: $55 \mathrm{~mm}$, underpressure: $0.04 \mathrm{MPa}$, excitation frequency: $0.02 \mathrm{~Hz}$ )

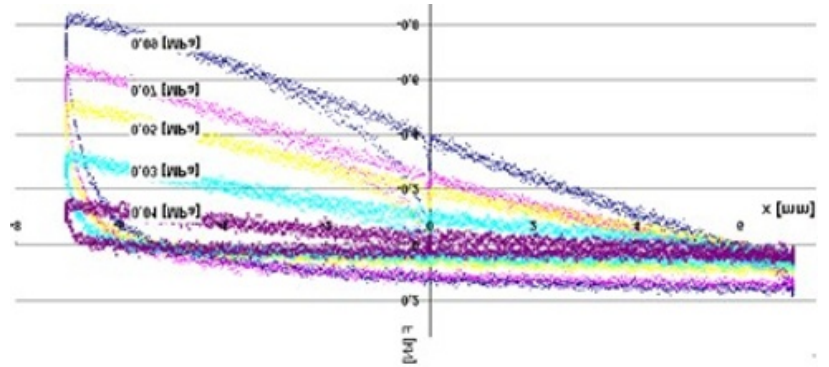

Fig. 6. The impact of the underpressure on the dissipative characteristics of VPP samples 


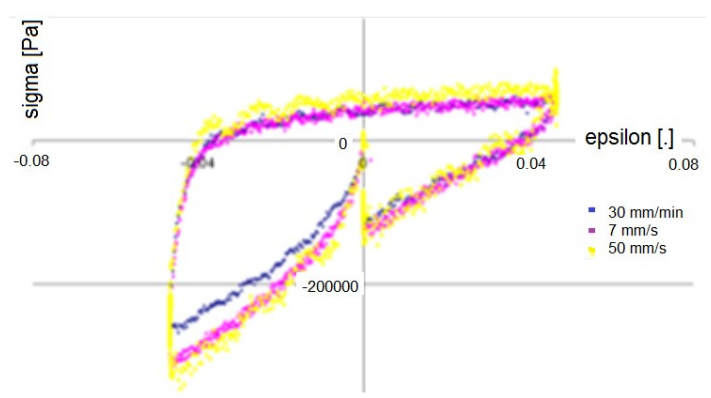

Fig. 7. The influence of kinematic deformation velocity

For the higher values of excitation frequency, the increase in ability of energy dissipation for VPP is observed. The description of viscous phenomena for this type of materials is not clearly understood yet. It should be remembered that investigated special granular structures exhibit a much higher degree of non-linearities than classical structural materials such as metals or polymers. In addition, the strongly non-linear effect of the underpressure parameter makes it difficult to analyze the impact of strain rate on the behavior of the examined conglomerates. An additional operational problem of special granular structures is the socalled 'scale effect'. Smaller volume samples exhibit different mechanical (rheological) properties than their larger equivalents. The problem of estimating the scale effect in granulated cores is very complex. More information about this phenomenon can be found in [10].To conclude the experimental part of the work, it is worth mentioning to the complexity of phenomena occurring in Vacuum Packed Particles. The problematic nature of their commercial use in engineering applications is complicated by the non-linear influence of the control factor - underpressure. Before starting the designing process of controllable VPP damper prototype it is necessary to overcome the basic problems encountered during the implementation of the basic research program.

\section{The concept of controllable VPP linear damper}

\subsection{The concept of controllable VPP linear damper}

The idea of designing a prototype of a granular damper with controlled dissipative properties was taken from the authors previous experience gained during research on MR dampers. Although MR fluids are one of the more commercialized intelligent materials, their use in engineering applications still poses some difficulties [11]. They can be divided into two main groups: technological and material.

In the group of technological problems one should mention the selection of appropriate construction materials for a given MR device, which physical characteristics must meet strictly defined assumptions. Magnetorheological fluids change their properties under the influence of an externally applied magnetic field [11]. Materials used for the construction of devices working on their basis should provide, among others the possibility of eliminating the phenomenon of residual magnetism - characteristic of many metals commonly used in machine designing.

The second group of commonly encountered difficulties is the use of the MR fluid itself. Such a material is generally a colloidal suspension of ferromagnetic particles in a carrier liquid. This fact means that when analyzing its mechanical properties, it is not 
possible to use equations taken from classical fluid mechanics. The nowadays observed rapid development of material engineering did not fully eliminate the phenomenon of sedimentation of solid particles at the bottom of a container filled with MR liquid. Another not fully understood problem is the influence of temperature on the properties of the MR liquid. One of the biggest challenges in more complete commercialization of MR devices in everyday engineering applications is the need to use advanced controllers. The necessity to analyze various external signals in real time requires the use of complicated and thus expensive electronic devices. In this short introduction to this chapter, only the outline of potential problems encountered in the construction of devices equipped with an MR device is outlined. Their entirety caused that the authors considered it reasonable to propose a prototype construction of a damper with a granulated core.

\subsection{VPP damper elements}

The main advantage of the proposed solution are economic aspects. The granular material used in the construction of the damper is much cheaper than the corresponding MR fluid. In addition, in the place of a complex electronic controller, in the investigated case, there is a relatively inexpensive vacuum pump. One of the negative features of special granular structures observed in operational conditions (with cyclic loading typical of the damper operation) is the tendency to local agglomeration of the working medium. This leads to a situation in which, after a certain number of working cycles, a kind of necking in the sample arises, showing similarities to the neck, observed with uniaxial tension tests of cylindrical metal samples. To eliminate the previously mentioned phenomenon, a compensating space in which the specially designed spring was placed was provided in the prototype of the granular VPP damper. Through a specially shaped disc, it eliminates the situation in which a plastic encapsulation becomes a working element (Fig. 8). While internal underpressure has a major impact on the global mechanical (dissipative) properties of the granular device, it is very important to maintain a constant and homogeneous pressure value inside the structure. In the proposed VPP damper prototype, a perforated soft rubber tube, placed axially inside the core of the device, was proposed. The end of the tube is a valve that allows the vacuum pump to be connected to the device (Fig. 9). The only task of the soft plastomer encapsulation surrounding the loose granules is to ensure air tightness. The proposed solution eliminates the possibility of it transmitting tensile stresses. Such a treatment was made possible by providing pre-deformation of the envelope in comparison to the working length of the granular core. In this particular case the additional soft spring having neglected stiffness was proposed to ruck the external envelope. The main spring is an element ensuring the return of the deformed granular core to its original position. Its stiffness is selected so that at the highest negative pressure (close to the technical vacuum) it is possible to stretch the pre-deformed conglomerate.

A schematic illustration of the prototype of the granular damper is shown in Fig. 10 


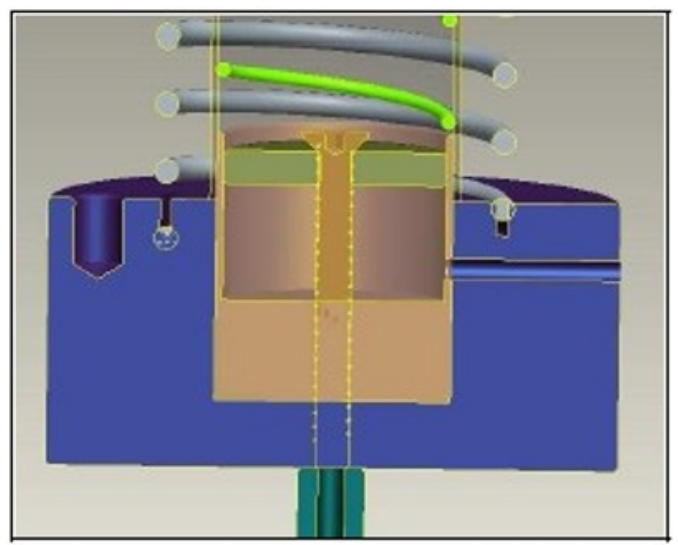

Fig. 8. The lower base of the granular damper prototype with the special chamber minimizing the packing effect of granular media

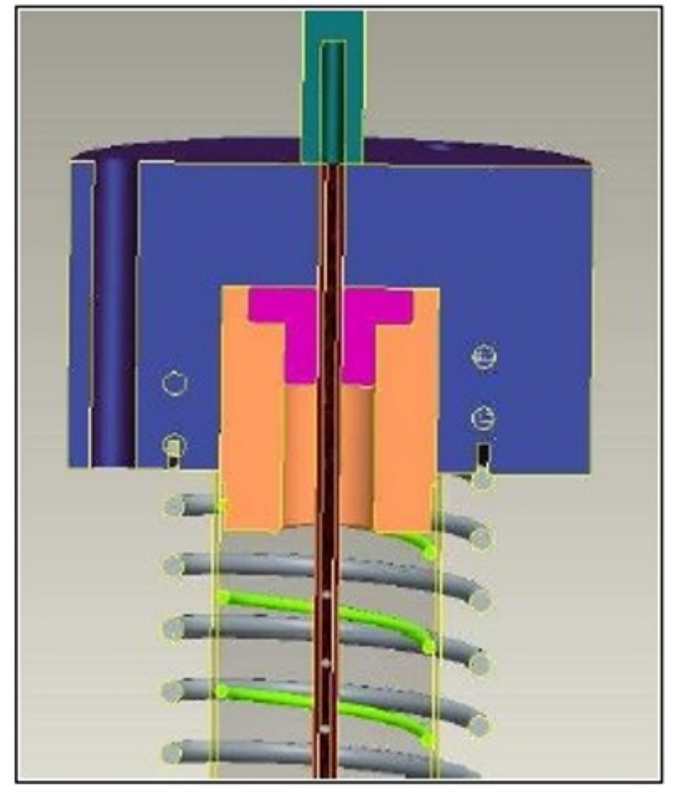

Fig. 9. The upper base of the granular damper including the valve and the rubber element ensuring a homogeneous value of the partial vacuum

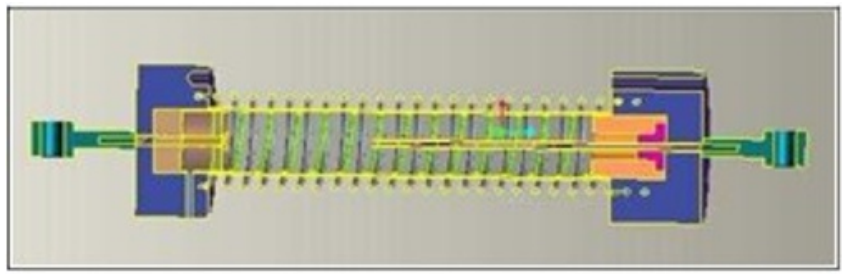

Fig. 10. An overview of the prototype of a controlled granular VPP damper 


\section{Experimental results for the controllable VPP damper}

In this section typical experimental data acquired for the discussed VPP controllable damper prototype are presented and discussed. The tests were performed at the same experimental stand as previously mentioned in the case of granular cores. In the laboratory tests four excitation frequencies $(1.5 ; 2.5 ; 5$ and $8 \mathrm{~Hz})$ and various underpressure values were taken into consideration. The main objective of laboratory tests was to reveal the possibility of controlling the dissipative properties of proposed device by the underpressure factor. In Fig. 11 experimental data recorded for the damper working with a lowest underpressure value (atmospheric pressure) are depicted. Analyzing the data it can be observed that the impact of the excitation frequency value, in the contrary to pure granular cores (Fig. 7), is negligible in the damper construction. The shape of the recorded dissipative characteristic is quite symmetric. Such a behavior is rapidly changing when the value of partial vacuum inside the system is increased (Figs. 12-14). The higher underpressure value generates unsymmetrical damping characteristics similarly like in the previously discussed case of pure granular cores.

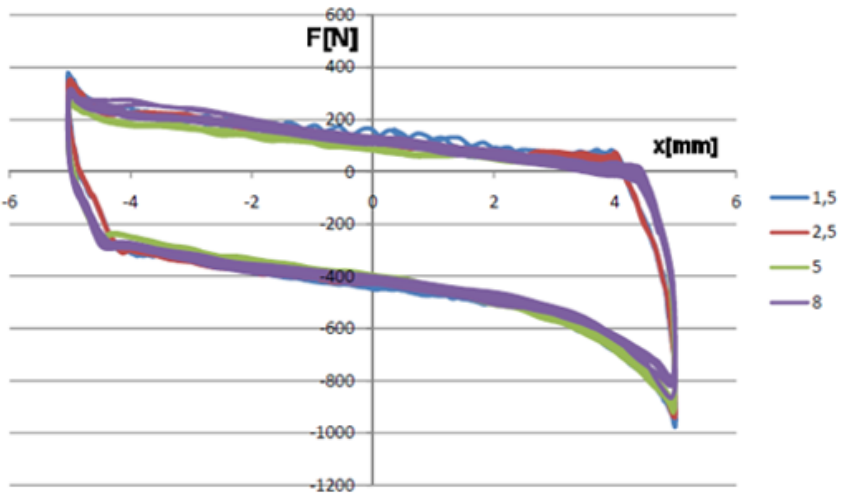

Fig. 11. Experimental results for VPP controllable damper; underpressure $=$ const. $(0 \mathrm{MPa})$; various excitation frequencies $(1.5 ; 2.5 ; 5 ; 8 \mathrm{~Hz})$

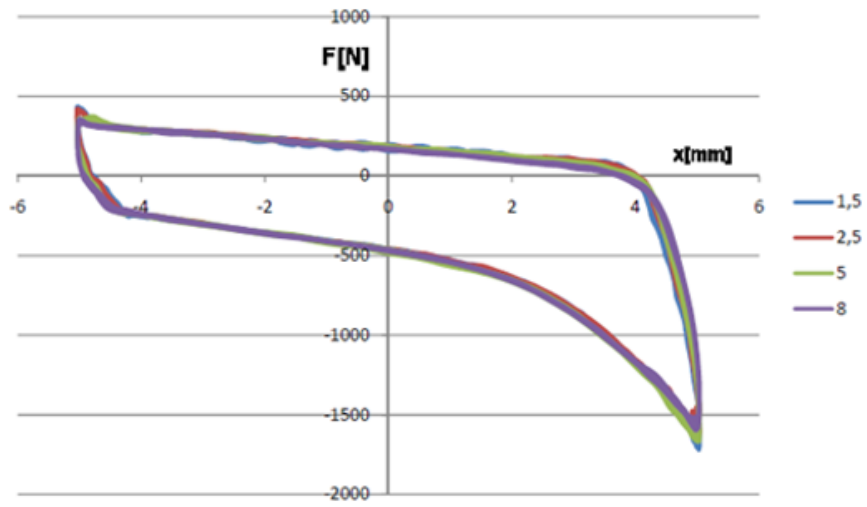

Fig. 12. Experimental results for VPP controllable damper; underpressure $=$ const. $(0.03 \mathrm{MPa})$; various excitation frequencies $(1.5 ; 2.5 ; 5 ; 8 \mathrm{~Hz})$ 


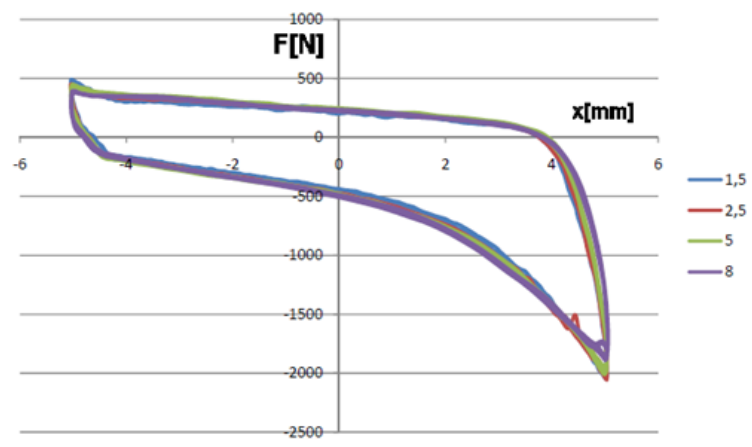

Fig. 13. Experimental results for VPP controllable damper; underpressure $=$ const. $(0.06 \mathrm{MPa})$; various excitation frequencies $(1.5 ; 2.5 ; 5 ; 8 \mathrm{~Hz})$

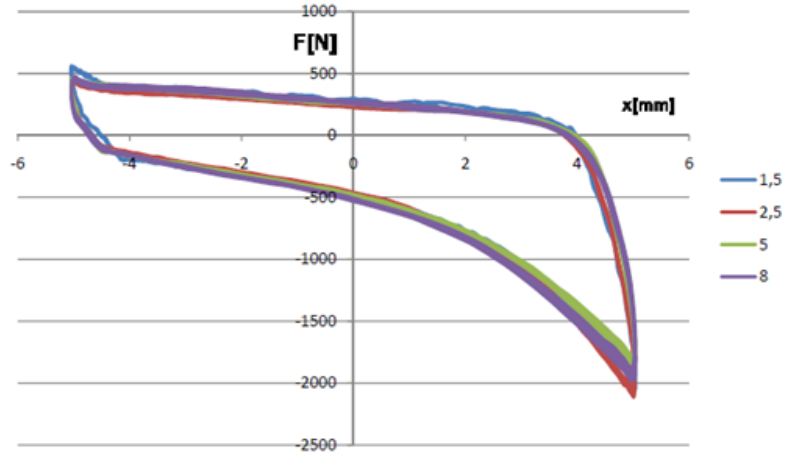

Fig. 14. Experimental results for VPP controllable damper; underpressure $=$ const. $(0.08 \mathrm{MPa})$; various excitation frequencies $(1.5 ; 2.5 ; 5 ; 8 \mathrm{~Hz})$

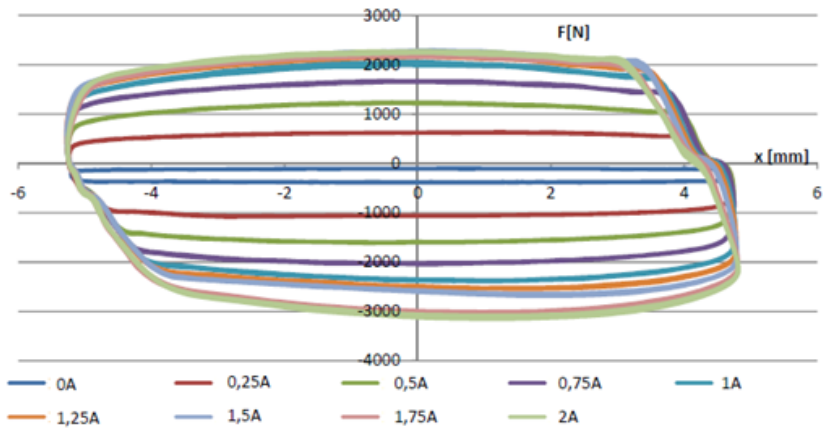

Fig. 15. Experimental results for Lord Co. MR damper; excitation frequency = const $(1.5 \mathrm{HZ})$; various current intensities $(0 ; 0.25 ; 0.5 ; 0.75 ; 1.0 ; 1.25 ; 1.5 ; 1.75 ; 2.0 \mathrm{~A})$

Moreover, higher compression forces are noticed for the increased values of partial vacuum. Such phenomenon confirms the possibility of controlling the damping properties of VPP absorbers. In Fig. 15 exemplary experimental results obtained for Lord RD-8041-1 MR damper are presented (Zalewski et al. 2014). The same triangular excitation rule with $1.5 \mathrm{HZ}$ frequency was taken into account in the laboratory tests. Applied in experiments various current intensity values revealed the excellent adaptive properties of the most popular on the market MR device. Increasing the current intensity value, the MR device improves its damping properties more than 20 times. Additionally observed in Fig. 15 characteristics are 
symmetrical. Such a shape of force-displacement diagram is commonly eligible in popular engineering applications.

\section{Conclusions}

In comparison to commercially available dampers and shock absorbers with variable dissipative properties, the proposed granular VPP damper has many advantages. The main advantage is the mentioned economic aspect. In addition, the granular device appears to be less sensitive to temperature changes compared to the analogous MR damper. Another advantage of the proposed solution is its lower sensitivity to pollution. In addition, in the case of granulated conglomerates, the problem of tightness is automatically eliminated. The basic limitation of the VPP prototype is its characteristics. One should remember about its asymmetric shape. However, in many engineering applications, this potential defect becomes an advantage. Often the device's constructor depends on effective attenuation of vibrations in only one undesirable direction. In this case, the proposed prototype solution seems to be optimal, especially when the user of the device is able to control its dissipative properties by means of a convenient parameter such as underpressure.

\section{References}

1. Bartkowski, P., Zalewski, R. (2018). A concept of smart multiaxial impact damper made of vacuum packed particles. In MATEC Web of Conferences, 157, page 05001. EDP Sciences

2. Brown, E., Rodenberg, N., Amend, J., Mozeika, A., Steltz, E., Zakin, M. R., Lipson, H., and Jaeger, H. M. (2010). Universal robotic gripper based on the jamming of granular material. Proceedings of the National Academy of Sciences, 107(44), 18809-18814

3. Zalewski, R., Szmidt, T., (2014). Application of Special Granular Structures for semiactive damping of lateral beam vibrations. Engineering Structures 65, 13-20

4. Bajkowski, J.M., Dyniewicz, B., Bajer, C.I., (2015). Damping properties of a beam with vacuum-packed granular damper. Journal of Sound and Vibration, 341, 74-85

5. Loeve, A. J., van de Ven, O. S., Vogel, J. G., Breedveld, P., and Dankelman, J. (2010). Vacuum packed particles as flexible endoscope guides with controllable rigidity. Granular matter, 12(6), 543-554

6. Pyrz, M., Zalewski, R. (2010). Modeling of granular media submitted to internal underpressure. Mech. Res. Commun., 37, 2: 141-144

7. Zalewski, R., Chodkiewicz, P., (2014). Gubanov model for vacuum packed particles. Mechatronics 2013: 57-63. Springer

8. Zalewski R., Shillor M., Chodkiewicz P., (2016). Vibrations of a mass-spring system using a granular-material damper. Applied Mathematical Modelling, 40: 8033-8047

9. Szmidt, T., Zalewski, R., (2014). Inertially excited beam vibrations damped by vacuum packed particles. Smart Materials and Structures, 23, 10, 105026 (9pp)

10. Zalewski, R., (2010). Constitutive model for special granular structures. Int. J. NonLinear Mech., 45, 3: 279-285

11. Makowski, M., Knap, L., (2018). Investigation of an off-road vehicle equipped with magnetorheological dampers. Advances in Mechanical Engineering, 10, 5: 1-11 